\title{
Percutaneous coronary intervention using a mechanical circulatory support system with an Impella centrifugal pump device combined with subsequent cryoablation for atrial fibrillation
}

\author{
Anna Winnicka-Zielińska* ${ }^{1 *}$ Bogdan Musielak ${ }^{1,2 *}$, Jan Budzianowski1 ${ }^{1,}(0$, \\ Jarosław Hiczkiewicz ${ }^{1,2}$, Paweł Burchardt ${ }^{3,4}$ \\ ${ }^{1}$ Clinical Department of Cardiology, Nowa Sol Multidisciplinary Hospital, Nowa Sol, Poland \\ ${ }^{2}$ Collegium Medicum, University of Zielona Gora, Poland \\ ${ }^{3}$ Department of Hypertensiology, Angiology and Internal Medicine, \\ Poznan University of Medical Sciences, Poznan, Poland \\ ${ }^{4}$ Department of Cardiology, J. Strus Hospital, Poznan, Poland
}

A 63-year-old man was admitted to an intensive care unit due to non-ST-segment elevation myocardial infarction. He had a history of hypertension, diabetes, coronary artery disease (CAD) and underwent percutaneous coronary intervention of the left circumflex artery and radiofrequency ablation of tricuspid isthmus in 2009 due to a persistent atrial flutter. Transthoracic echocardiography showed a severely reduced left ventricular ejection fraction (LVEF 25\%) and severe mitral regurgitation. The patient was qualified for urgent coronarography which revealed advanced multivessel CAD (Fig. 1A, B).

The Heart Team decided to perform rotablation of the left anterior descending artery with mechanical circulatory support using an Impella centrifugal pump (CP) due to the high risk associated with a surgical procedure. The Impella $\mathrm{CP}$ was introduced into the left ventricle through the left femoral artery obtaining $4.0 \mathrm{~L} / \mathrm{min}$ flow. The procedure was effective and the blood flow marked as grade 3 in the Thrombolysis in Myocardial Infarction (TIMI) system was achieved (Fig. 1C).

Three weeks following his discharge, he experienced an episode of symptomatic paroxysmal atrial fibrillation which led to exacerbation of heart failure (class III according to the New York Heart Association [NYHA] system). The decision was made to urgently perform cryoablation of pulmonary veins (Arctic Front Advance $2^{\text {nd }}$ generation $28 \mathrm{~mm}$ ) (Fig. 1D). Further hospitalization was carried out without complications or recurring arrhythmia. The patient was discharged in a stable condition (class II of NYHA).

Mechanical circulatory support with an Impella $\mathrm{CP}$ device allows for percutaneous procedures in patients with a severely reduced LVEF. It was proven that pulmonary vein isolation in patients with heart failure with reduced ejection fraction was a safe and feasible treatment modality.

Conflict of interest: None declared

Address for correspondence: Jan Budzianowski, MD, PhD, Collegium Medicum, University of Zielona Gora, ul. Zyty 28, 65-046 Zielona Góra, Poland, tel: +48 683882 103, e-mail: jbudzianowski@uz.zgora.pl

Received: 21.02.2021 Accepted: 6.06.2021

*Equal contribution

This article is available in open access under Creative Common Attribution-Non-Commercial-No Derivatives 4.0 International (CC BY-NC-ND 4.0) license, allowing to download articles and share them with others as long as they credit the authors and the publisher, but without permission to change them in any way or use them commercially. 


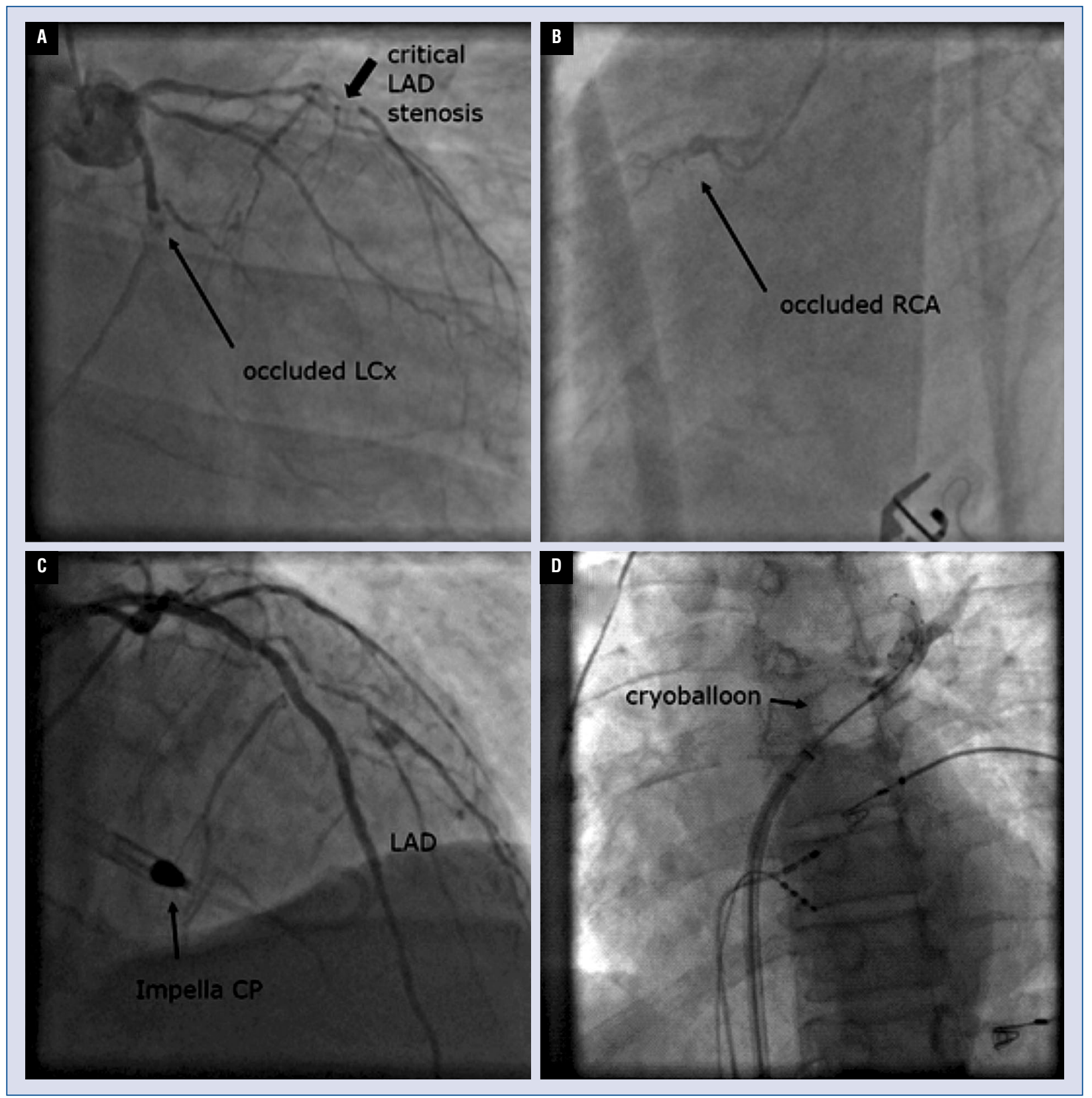

Figure 1. A, B. Initial coronarography: significant stenosis of the $6 / 7$ segment and critical stenosis of the left anterior descending artery (LAD) segment 8 , a chronic total occlusion of the left circumflex artery (LCx) in the stent, occlusion of marginal branch 1, a critically stenosed intermediate branch and chronic total occlusion of the right coronary artery (RCA); C. The Impella centrifugal pump (CP) in left ventricle. The RCA was intubated through the right femoral access with an EBU 4.0 6FrS guide catheter. The BHW introducer was used for the LAD. Predilatation of critical stenoses was performed by means of $2.0 \times 15 \mathrm{~mm}$ and $3.0 \times 20 \mathrm{~mm}$ balloon catheters. Three Orsiro drug eluting stents $(2.75 \times 30 \mathrm{~mm}$, $3.0 \times 40 \mathrm{~mm}, 3.0 \times 22 \mathrm{~mm}$ ) were implanted without complications; D. During the cryoapplication in the upper left pulmonary vein, the sinus rhythm returned. 